\title{
Research on the Method of Multi-point Pressing Adjustment of Space Mechanism Driven by Measurement
}

\author{
Jinlong Zhao ${ }^{1,2, a}$, Chunzhen Ren ${ }^{1,2}$, Zhizhuo Cui ${ }^{3}$ and Fuzhou Du ${ }^{3}$ \\ ${ }^{1}$ Beijing Institute of Spacecraft Environment Engineering, Beijing, China \\ ${ }^{2}$ Beijing Engineering Research Center of the Intelligent Assembly Technologyand Equipment for Aerospace Product, Beijing, China \\ ${ }^{3}$ Beihang University, Beijing, China
}

\begin{abstract}
In order to solve the problems of difficult assembly and adjustment and poor operation accessibility in the assembly process of multi-point pressure-type large-scale equipment on spacecraft, this paper presents a method of multi-point compact assembly of space mechanism driven by measurement. The method establishes the geometric constraint mathematical model of the equipment pressing mechanism, proposes the assembly coordinate system based on the mounting hole and the compensation method based on the rigid body transformation, and develops the relevant software according to the method. This method has been verified during the installation of the equipment and has achieved good results, which can be used as a reference for other digital assembly methods of aerospace equipment.
\end{abstract}

\section{Introduction}

In recent years, digital assembly technology has flourished, and because of its high precision, high efficiency and low risk, it is gradually replacing traditional assembly technology. At present, for the assembly features of different scale assemblies, the corresponding digital assembly technology can be used for efficient assembly operations, such as small-scale robot shaft hole assembly ${ }^{[1]}$, large-scale wall panel flexible assembly ${ }^{[2]}$, large parts digitization docking ${ }^{[3]}$ and so on.

In the aerospace field, with the continuous development of manned space technology, the various types of equipment carried on the spacecraft have been greatly improved in terms of quantity, scale and functionality. Among them, there is a large-scale multipoint compression type large cabin. The equipment has the characteristics of large mass and size, multiple degrees of freedom, multiple joints, and uneven mass distribution. In order to ensure that the equipment does not damage during the launching process, the connection mode between the equipment and the spacecraft cabin is designed as a multi-point compression self-locking connection ${ }^{[4-5]}$. The self-locking compression mechanism locks the device in the ground assembly and launching state, and unlocks and releases the device after entering the rail, so that it enters the normal working state ${ }^{[6]}$. At present, the typical multi-point compression large-scale outboard equipment includes space manipulator ${ }^{[7-9]}$, solar battery wing, etc.

Multi-point compression equipment assembly generally uses the position and attitude of the pressing mechanism that the gasket adjustment device cooperates with the cabin. The pressing mechanism in the assembly is distributed on different planes, the relative spatial position is complex, and the installation precision of each pressing mechanism is high. Secondly, due to the large scale of the assembly itself, the operator can't enter the inner side of the mating surface for operation, and can only be assembled by repeated trial and adjustment, so the assembly efficiency is low and some risks are always exist. In addition, there is a problem of poor accessibility during the installation and alignment of the equipment due to the relatively complicated layout position of the equipment pressing mechanism. Therefore, it is necessary to adopt a digital measurement-based scheme to guide the assembly of the pressing mechanism, and reduce the repeated cycle of the trial-and-adjustment during assembly, so assembly at one time can be achieved and the assembly efficiency and ensuring assembly quality are improved.

Aiming at the structural characteristics and assembly requirements of a certain type of multi-point pressuretype large-scale equipment on manned spacecraft, this paper proposes a measurement-driven multi-point compression fitting method to solve the problems of difficult installation and adjustment, poor accessibility and low efficiency exist in the loading process of this kind of equipment.

\section{Multi-point compression assembly}

\subsection{The structure of the pressing mechanism}

\footnotetext{
a Corresponding author: zhaojinlongcasc@163.com
} 
Compression connection is a unique form of fastening connection, and a single compression mechanism is divided into an upper pressing seat and a lower pressing seat (also called release nut). Under normal circumstances, the upper pressing seat is self-contained, and the lower pressing seat is connected to the mounting surface of the device. When the equipment is installed, it is necessary to precisely fit the plurality of upper pressing seats on the equipment and the corresponding plurality of lower pressing seats on the mounting surface, and then perform locking by the locking device between the pressing seats. The basic structure of the pressing mechanism is shown in Figure 1 (the locking device is not shown).

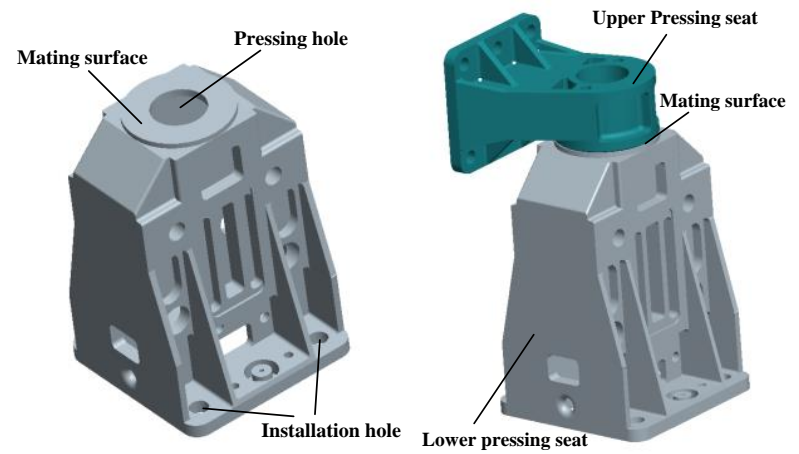

Figure 1. Pressing mechanism and connection diagram

\subsection{Assembly requirements for the compression mechanism}

The assembly form of the pressing mechanism is the cooperation of the two end faces of the upper and lower pressing seats, and the assembly requirements are mainly embodied in the gap of the mating surface and the relative position of the upper and lower circular holes. According to the assembly requirements, the corresponding mathematical relationship can be obtained, as shown in formulas (1) to (3), and the coordination relationship is shown in Figure 2.

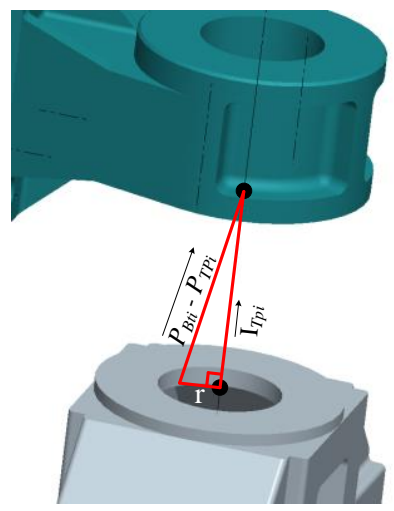

Figure 2. Schematic diagram of the cooperation mechanism of the pressing mechanism

$$
\begin{gathered}
\left|\overrightarrow{P_{B t l}-P_{T p l}} \cdot \overrightarrow{I_{T p l}}\right|<c \\
\sqrt{{\overrightarrow{P_{B t l}-P_{T p l}}}^{2}-\overline{P_{B t l}-P_{T p l}} \cdot \vec{I}_{T p l}^{2}}<r
\end{gathered}
$$

$$
\angle\left(\overrightarrow{I_{T p l}}-\overrightarrow{I_{B t \imath}}\right)<\theta
$$

In the formula, $P_{T p i}$ and $P_{B t i}$ are the coordinates of the hole center of the pressing surface of upper and lower pressing seats on the mating surface, $r$ is the coaxiality requirement of the fitting in the assembly, and is indirectly expressed by the deviation of the center position of the mating surface, and $c$ is the clearance requirement of assembly fit, $\theta$ is the axial angle requirement for the clamping mechanism, $\overrightarrow{I_{T p}}$ and $\overrightarrow{I_{B t \imath}}$ are the normal vectors of the mating surfaces.

\subsection{Working condition analysis of multi-point pressing assembly}

Under normal circumstances, when multi-point pressuretype large equipment is assembled, the pressing mechanism is distributed in multi points and different planes in space, and the matching form is shown in Figure 3.

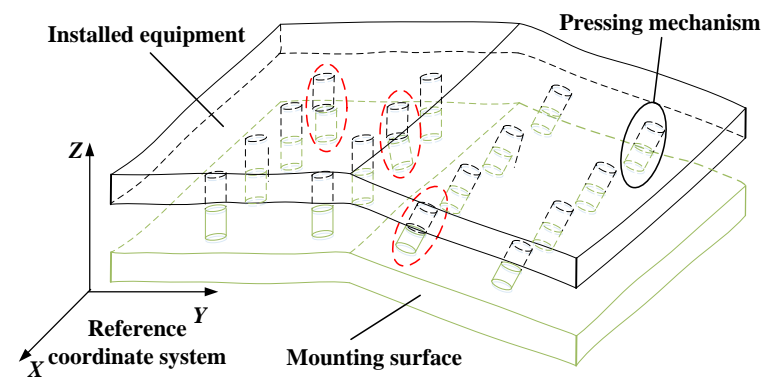

Figure 3. Pressing mechanism and connection diagram

When the lower pressing seat of the pressing mechanism is mounted on the equipment mounting surface, it is connected to the mounting surface through 4 bolt holes. Each mounting hole has a $\Phi 1 \mathrm{~mm}$ translatable interval for adjusting the position, and the height and inclination can be adjusted properly by the gasket of 4 mounting holes, as shown in Figure 4.

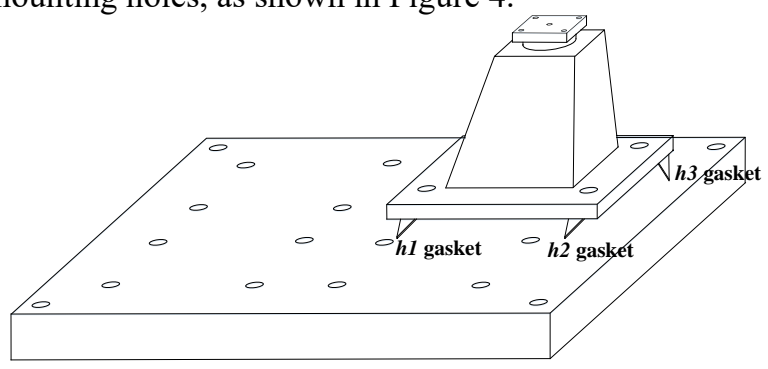

Figure 4. Pressing seat installation and adjustment diagram

Since the lower pressing seat of each pressing mechanism is flexible and adjustable, it is difficult to ensure that the mating surfaces of the upper and lower pressing seats of each pressing mechanism can meet the matching requirements during the assembly of the whole equipment, and it is easy to have partial or most of the mating surfaces pressing mechanism offset, skew, clearance and other situations, which eventually lead to the failure of the matching, stress concentration and other problems, and ultimately affect assembly quality. 
In general, multi-point pressure-type equipment is mostly multi-faceted and is usually fixed to a specific tooling (called a simulated wall) before delivery. All the pressing mechanisms on the tooling are consistent with the cabin and the rigidity is good, which can well maintain the posture state of the device. The installation state of the equipment on the simulated wall is the best installation state, and the fitting tolerance of each pressing device is satisfactory. According to the requirements, all the pressing mechanisms (lower pressing seats) on the simulated wall must be removed and installed on the cabin, and then the pressing mechanism on the cabin is adjusted during the loading process. The posture state is consistent with the tooling state, thereby realizing the installation of the device on the cabin.

\section{Transfer scheme of benchmark based on mounting holes}

For ease of description, the multi-point pressure-type large equipment for loading is classified as a docking assembly, and the mounting surface of the cabin is referred to as a reference assembly, and the simulated wall is referred to as a tooling.

In the digital assembly, the transfer of the reference is carried out step by step from the actual object. The reference coordinate system quoted in the measurement is an important part of the reference transmission, which directly affects the model posture and state changes observed by the operator in the three-dimensional computer space. In the process of benchmark transmission, it is necessary to ensure the consistency of the benchmark transmission and reduce the error of the reference transmission, so as to effectively reduce the deviation of the attitude caused by the deviation of the coordinate system.

In the process of digital assembly of docking assembly on spacecraft, the transfer of datum usually involves three coordinate systems: design coordinate system, tooling coordinate system and datum assembly coordinate system (assembly coordinate system).

The design coordinate system is the reference coordinate system used in the design process of the equipment. Usually, one of the key point of the product is taken as the origin of the coordinate system, and the axis of symmetry or the central axis is taken as the axial direction, and the horizontal plane is parallel with the axis to establish another axial direction. The right hand rule establishes the third axis. The tooling coordinate system is the coordinate system represented by the specially processed measuring hole position and its calibration coordinates after the tooling design is completed and processed. In general, the design coordinate system and tooling coordinate system of docking assembly have been determined in the stage of design and production department assembly, and relevant measurement work has been completed by the designer. In the loading phase, the tooling coordinate hole is marked on the tooling. The coordinate system consistent with the design coordinate system can be quickly obtained on the tooling, and only the coordinate system of the reference assembly needs to be determined later.

However, in most cases, the reference assembly is limited by its own functional and structural requirements, and it is not allowed to directly manufacture the dedicated reference hole corresponding to the tooling as the established value of the reference coordinate system, so there is no reference hole position with the same coordinate system on the tooling, which make it impossible to establish a coordinate system consistent with the tooling coordinate system during assembly, so the transfer of the reference cannot be smoothly performed.

In order to solve the above problems, considering that the mounting holes of the pressing mechanism are provided on the tooling and the reference assembly, and the hole position layout is consistent with the topological relationship, the installation hole of the pressing mechanism at some specific positions is taken as the reference hole position established in the coordinate system. So the reference coordinate system on the tooling can be transferred to the reference assembly (i.e. the cabin) by this reference transfer method. The reference system transfer process is shown in Figure 5, and the partial compression mechanism mounting holes in the figure have been omitted.

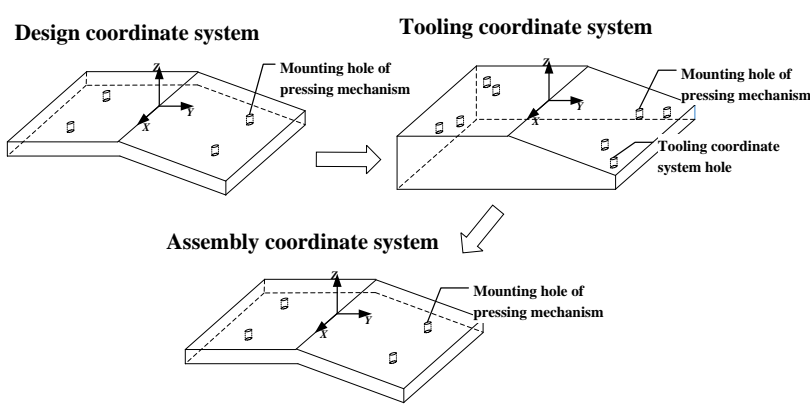

Figure 5. Coordinate system transfer process

According to the existing working conditions, there are three options for selecting the measured value of the mounting hole of the pressing mechanism as follows:

- Select the design theoretical value given by the design coordinate system and the coordinate system transfer route is from the design coordinate system to the assembly coordinate system

- Select the measured value obtained by the designer according to the physical measurement and verification. This scheme is an improvement of the first scheme;

- Select the measured value of the corresponding mounting hole on the tooling under the current working condition environment. The transfer route of coordinate system is from the design coordinate system to the tooling coordinate system and then to the assembly coordinate system, which requires two times of datum transfer process.

Because the docking assembly is in the best fit with the tooling after undergoing transportation, hoisting and pre-testing, it is impossible to objectively agree with the theoretical value of the design. Therefore, only the mounting state of the pressing mechanism on the tooling can accurately reflect the posture of the pressing 
mechanism on the docking assembly, and the coordinate system on the tooling can best reflect the state of the docking assembly. Therefore, the third option is the best choice.

In the actual assembly, after the butt assembly is separated from the tooling, the lower pressing seat is taken out one by one and restored to the reference assembly according to the tooling, so that the optimal reference assembly can be matched with the docking assembly. In selecting the mounting hole of the pressing mechanism, a total of 16 mounting holes covering the outer edges of the main mounting surface in four pressing mechanisms can be selected as the coordinate system reference point.

\section{Compensation calculation method}

According to the structural characteristics of the lower pressing seat of the pressing mechanism, the measuring aid is used to measure the posture of the measuring device. The measuring aid has 5 measurable hole positions, wherein the central hole position is the spare measuring hole position, and only 4 holes are needed for measurement, as shown in Figure 6.

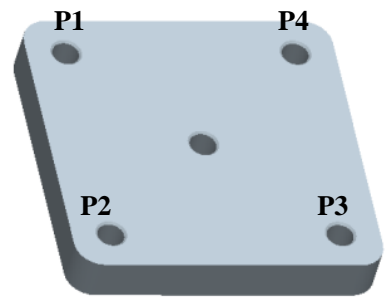

(a) Front

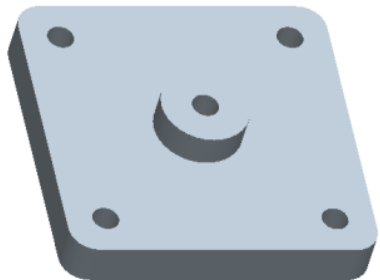

(b) Back
Figure 6. Schematic diagram of the measuring aid

According to formula (4), the position and normal vector of the center point of the measuring aid can be calculated, and the same measuring aid can be used to measure the pressing mechanism to avoid systematic errors caused by using different measuring aids.

$$
\left\{\begin{array}{c}
\overrightarrow{O_{l}}=\frac{\overrightarrow{\left(P_{3}-P_{1}\right)} \times \overline{\left(P_{4}-P_{2}\right)}}{\mid \overline{\left(P_{3}-P_{1}\right)} \times\left(P_{4}-P_{2}\right)} \\
P_{i}=\frac{1}{4} \sum_{j=1}^{4} P_{j}-L \cdot \overrightarrow{O_{l}}
\end{array}\right.
$$

We can restore the target attitude parameter on the reference assembly, which is measured by the measuring aid in the tooling coordinate system. If we calculate the amount of gasket and the amount of translation at the installation hole according to the geometric dimension of the compression mechanism, we need to consider the installation direction of the compression mechanism and other conditions, which will make the calculation process very complicated. Therefore, we can use the method based on the change of rigid body to calculate, so that the four mounting holes of the pressing mechanism and the position and direction parameters of the measuring auxiliary center point calculated by formula (4) form a pyramid. Since the rotation of the measuring aid does not affect the position of the center point, the pyramid always remains relatively unchanged, as shown in Figure 7.

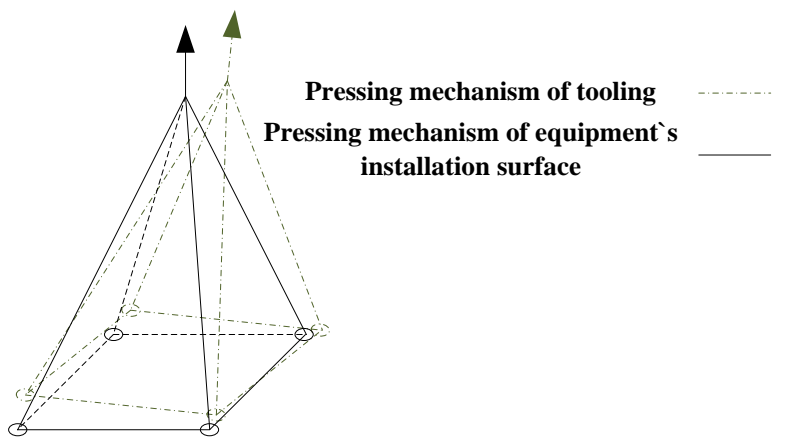

Figure 7. Pressing mechanism adjustment calculation diagram

Therefore, the calculation of the variation includes two parts, the direction vector rotation and the positional degree translation. The direction vector is solved by the Rodrigue rotation matrix, and the translation is solved by vector subtraction, as shown in equations (5) and (6).

$$
\begin{gathered}
\overrightarrow{O_{l}^{D}}=R \overrightarrow{O_{l}^{T}} \\
R=-\left(\cos (\theta) \vec{I}+(1-\cos (\theta)) r r^{T}+\sin (\theta)\left[\begin{array}{ccc}
0 & -r_{z} & -r_{y} \\
r_{x} & 0 & -r_{z} \\
-r_{y} & r_{x} & 0
\end{array}\right]\right)
\end{gathered}
$$

In the formula, $\overrightarrow{O_{l}^{D}}$ and $\overrightarrow{O_{l}^{\mathrm{T}}}$ are the measured direction vectors on the reference assembly and tooling respectively. $\theta$ is the angle between the two, and $r$ is the unit obtained by the cross-multiplication vector. After calculating the rotation matrix, rotate and transform the mounting hole of each pressing mechanism to obtain the new position of the point, then calculate the distance between the new position and the original position on the direction vector, and subtract the minimum gasket thickness together, so as to obtain the gasket thickness value of each mounting hole to compensate the rotation deviation. Calculate the position of the apex of the pyramid at this time, and subtract it from the position parameters measured by the tooling. The obtained amount is the translation amount. By projecting the calculation amount on the plane of the direction vector direction and the vertical direction vector respectively, the gap compensation amount and the translation compensation amount caused by the gap can be calculated.

\section{Multi-point compression assembly test}

The above theory is verified by the installation of a large multi-point pressure-type equipment on a manned spacecraft. The assembly process is shown in Figure 8 . 


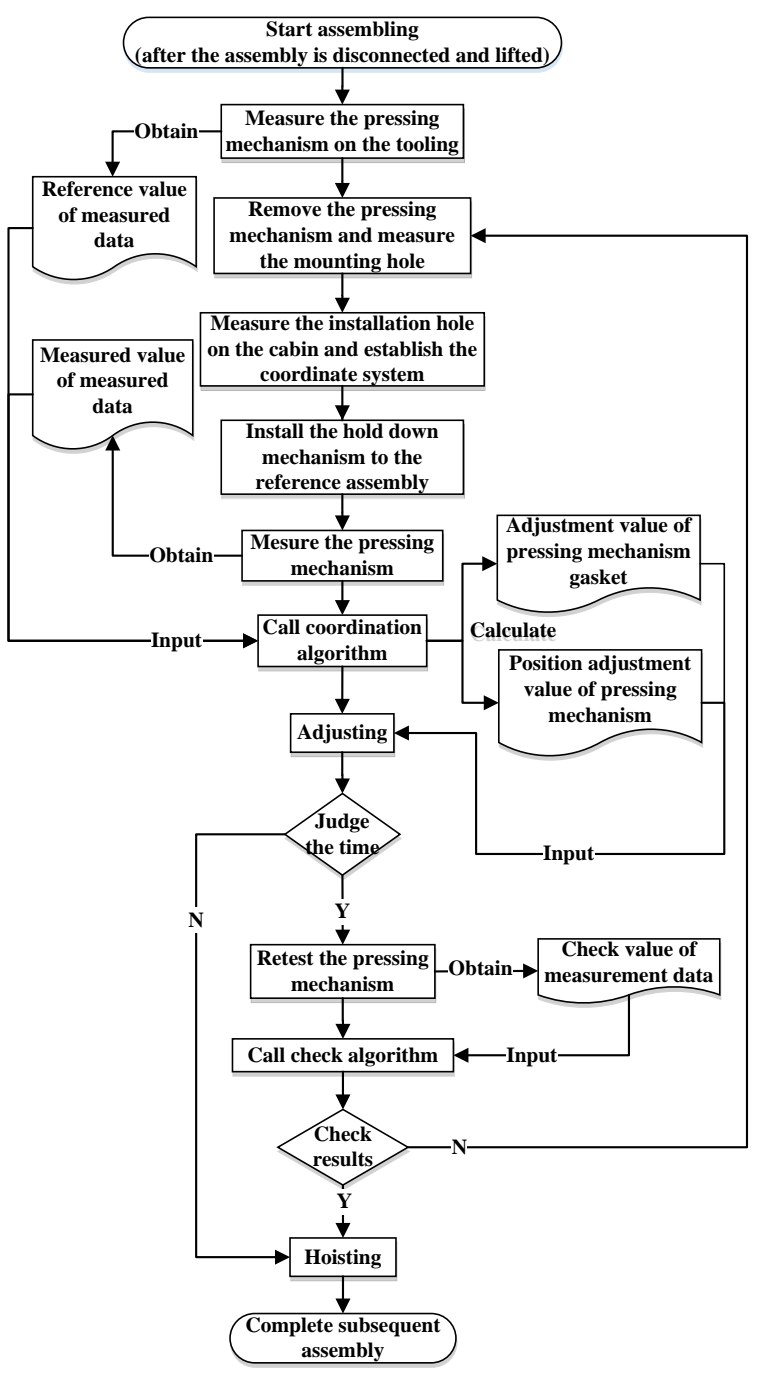

Figure 8. Multi-point pressing mechanism assembly process

Firstly, the tooling coordinate system is established according to the coordinate system calibration hole which is on the tooling, and the parameters of the pressing mechanism on the tooling are measured by the measuring aid. Then dismantle the pressing mechanism on the tooling, and measure the installation hole of the coordinate system to be established on the cabin. And Then measure the corresponding installation holes on the cabin and establish the coordinate system. After all the pressing mechanisms are installed, we can measure all the pressing mechanisms and calculate the compensation amount according to the calculation method of the adjustment amount, and adjust the position and posture one by one according to the compensation amount. Finally, retest and inspection are carried out.

For this assembly process, we developed the measurement and data analysis of multi-point pressing assembly coordination software to assist the assembly and adjustment process, and integrated the laser tracker measurement, data storage and management, data analysis, three-dimensional display, online assembly and adjustment oriented to the laser tracker and other functional modules. Part of the software operation interface is shown in Figure 9. The online adjustment is to ensure that the spatial position of the pressing mechanism can be instantly reacted. The tracking measurement mode of laser tracker is adopted, and the target ball is placed on the measuring aids to compare its deviation with the target value, so as to analyze whether the pressing mechanism is installed and adjusted successfully in real time.

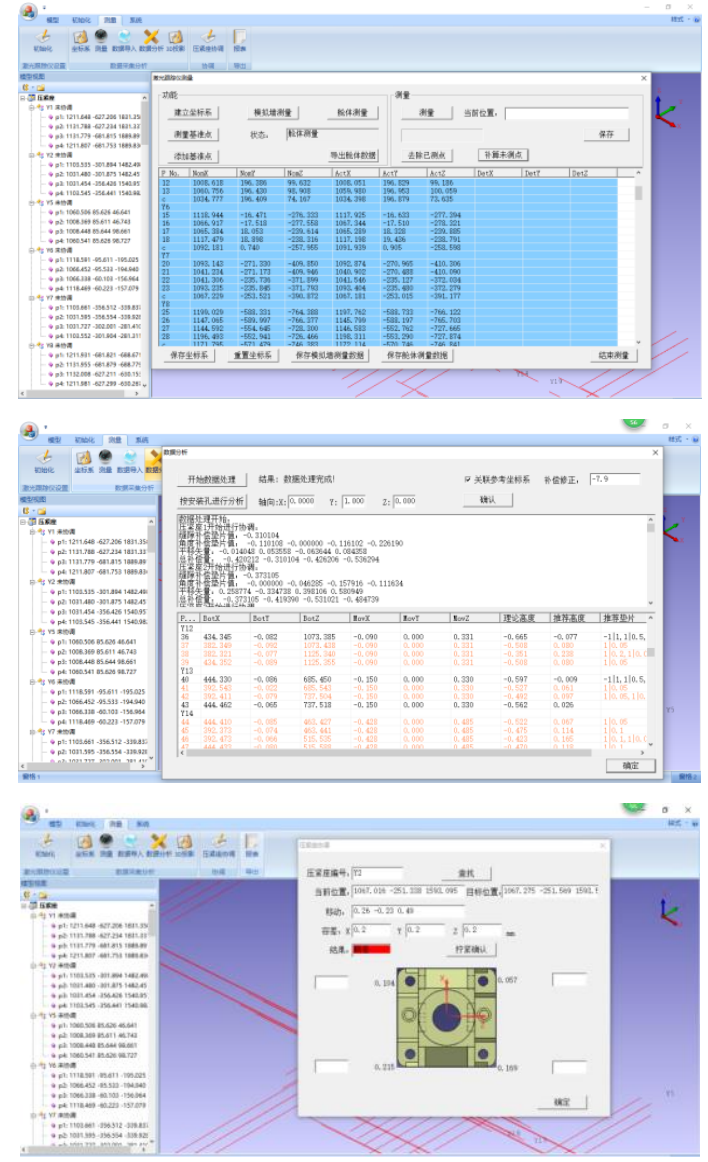

Figure 9. Multi-point compression coordination software interface

Table 1 shows the adjustments calculated by the software after the measurement of the equipment pressing mechanism. The equipment has a total of 41 sets of pressing mechanisms. Because of the huge amount of data, Table 1 only lists the adjustment of the pressing seats from Y1 to Y4. In the table, the compensation value of the gasket of the four hole positions of each pressing seat is divided into the theoretical value and the correction value. Considering that the compensation value of the hole position gasket of the pressing seat must be positive (that is, the pressing seat can only be padded upward, not downward), so the theoretical value is modified as a whole according to the topological relationship of the equipment pressing seat. The minimum negative value is modified to 0 , and the rest values are compensated accordingly by using the software. So the corrected gasket compensation value can be obtained finally.

The $\mathrm{X}$ and $\mathrm{Z}$ directions defined in the table are respectively the two directions of the pressing seat along the mounting plane, which can be adjusted online by 
software until the requirements are met. The Y direction

is the normal direction of the mounting surface of the

Table 1. Measurement results before the coordination of the pressing mechanism (Partial data) (Unit: $\mathrm{mm}$ )

\begin{tabular}{|c|c|c|c|c|c|c|}
\hline $\begin{array}{l}\text { No. of pressing } \\
\text { mechanism }\end{array}$ & $\begin{array}{l}\text { Translation } \\
\text { value in } X \\
\text { direction } \\
\end{array}$ & $\begin{array}{c}\text { Translation } \\
\text { value in Y } \\
\text { direction } \\
\end{array}$ & $\begin{array}{c}\text { Translation } \\
\text { value in } \mathbf{Z} \\
\text { direction } \\
\end{array}$ & $\begin{array}{c}\text { Hole position of } \\
\text { lower pressing } \\
\text { seat }\end{array}$ & $\begin{array}{c}\text { Theoretical } \\
\text { value of gasket } \\
\text { compensation }\end{array}$ & $\begin{array}{c}\text { Correction } \\
\text { value of gasket } \\
\text { compensation } \\
\end{array}$ \\
\hline \multirow{4}{*}{ Y1 } & \multirow{4}{*}{-0.4476} & \multirow{4}{*}{-0.2721} & \multirow{4}{*}{0.2916} & 1 & -0.5848 & 0.0960 \\
\hline & & & & 2 & -0.6696 & 0.0111 \\
\hline & & & & 3 & -0.6879 & 0.0072 \\
\hline & & & & 4 & -0.6026 & 0.0781 \\
\hline \multirow{4}{*}{ Y2 } & \multirow{4}{*}{0.4961} & \multirow{4}{*}{-0.2055} & \multirow{4}{*}{0.2205} & 1 & -0.3141 & 0.3667 \\
\hline & & & & 2 & -0.5083 & 0.1725 \\
\hline & & & & 3 & -0.5758 & 0.1050 \\
\hline & & & & 4 & -0.3828 & 0.2980 \\
\hline \multirow{4}{*}{ Y3 } & \multirow{4}{*}{0.3536} & \multirow{4}{*}{0} & \multirow{4}{*}{-0.1093} & 1 & 0.1426 & 0.8234 \\
\hline & & & & 2 & 0.2028 & 0.8836 \\
\hline & & & & 3 & 0.2236 & 0.9043 \\
\hline & & & & 4 & 0.1633 & 0.8441 \\
\hline \multirow{4}{*}{ Y4 } & \multirow{4}{*}{0.166} & \multirow{4}{*}{-0.0707} & \multirow{4}{*}{-0.0757} & 1 & -0.2003 & 0.4805 \\
\hline & & & & 2 & -0.1257 & 0.5550 \\
\hline & & & & 3 & -0.0760 & 0.6047 \\
\hline & & & & 4 & -0.1511 & 0.5296 \\
\hline$\ldots \ldots$ & $\ldots \ldots$ & $\ldots \ldots$ & $\ldots \ldots$ & $\ldots \ldots$ & $\ldots \ldots$ & $\ldots \ldots$ \\
\hline
\end{tabular}

Table 2. Retest results after compression of the pressing mechanism (Partial data) (Unit: mm)

\begin{tabular}{|c|c|c|c|c|c|c|}
\hline $\begin{array}{l}\text { No. of pressing } \\
\text { mechanism }\end{array}$ & $\begin{array}{c}\text { Translation } \\
\text { value in } X \\
\text { direction } \\
\end{array}$ & $\begin{array}{c}\text { Translation } \\
\text { value in Y } \\
\text { direction } \\
\end{array}$ & $\begin{array}{c}\text { Translation } \\
\text { value in } \mathbf{Z} \\
\text { direction } \\
\end{array}$ & $\begin{array}{c}\text { Hole position of } \\
\text { lower pressing } \\
\text { seat }\end{array}$ & $\begin{array}{c}\text { Theoretical } \\
\text { value of gasket } \\
\text { compensation }\end{array}$ & $\begin{array}{c}\text { Correction } \\
\text { value of gasket } \\
\text { compensation } \\
\end{array}$ \\
\hline \multirow{4}{*}{ Y1 } & \multirow{4}{*}{-0.014} & \multirow{4}{*}{0.0536} & \multirow{4}{*}{-0.0636} & 1 & -0.4202 & 0.0899 \\
\hline & & & & 2 & -0.4101 & 0.1000 \\
\hline & & & & 3 & -0.4262 & 0.0839 \\
\hline & & & & 4 & -0.4363 & 0.0738 \\
\hline \multirow{4}{*}{$\mathrm{Y} 2$} & \multirow{4}{*}{-0.0155} & \multirow{4}{*}{-0.0082} & \multirow{4}{*}{0.0665} & 1 & -0.4661 & 0.044 \\
\hline & & & & 2 & -0.458 & 0.0521 \\
\hline & & & & 3 & -0.4359 & 0.0742 \\
\hline & & & & 4 & -0.4463 & 0.0638 \\
\hline \multirow{4}{*}{ Y3 } & \multirow{4}{*}{0.1332} & \multirow{4}{*}{0.0001} & \multirow{4}{*}{0.1701} & 1 & -0.4624 & 0.0477 \\
\hline & & & & 2 & -0.4973 & 0.0128 \\
\hline & & & & 3 & -0.4762 & 0.0339 \\
\hline & & & & 4 & -0.4412 & 0.0689 \\
\hline \multirow{4}{*}{ Y4 } & \multirow{4}{*}{-0.1068} & \multirow{4}{*}{0.0267} & \multirow{4}{*}{0.0740} & 1 & -0.5071 & 0.003 \\
\hline & & & & 2 & -0.4609 & 0.0492 \\
\hline & & & & 3 & -0.452 & 0.0581 \\
\hline & & & & 4 & -0.4981 & 0.012 \\
\hline$\ldots \ldots$ & $\ldots \ldots$ & $\ldots \ldots$ & $\ldots \ldots$ & $\ldots \ldots$ & $\ldots \ldots$ & $\ldots \ldots$ \\
\hline
\end{tabular}

pressing seat, and its translation adjustment is ultimately based on the gasket compensation value of the mounting hole, so it is not the basis for adjusting the posture of the pressing seat itself, which can be ignored.

According to the calculated translation value and the gasket compensation value, after all the lower pressing seats of the equipment is adjusted in position, the measuring aid and the laser tracker are used again to retest, and the retest results are shown in Table 2.
It can be seen from Table 2 that the positional deviation is controlled within $0.2 \mathrm{~mm}$ according to the previously adjusted compensation value, and the height deviation is controlled within $0.1 \mathrm{~mm}$ to meet the assembly requirements of the equipment. According to statistics, the entire installation cycle of the equipment is about 12 hours. Compared with the previous traditional adjustment time ( 24 hours), the efficiency is increased by $50 \%$ and the quality of installation and adjustment becomes more reliable by using this method. 


\section{Conclusion}

Aiming at the assembly process of multi-point pressuretype large-scale equipment on spacecraft, a method of multi-point compact assembly and adjustment based on digital measurement is proposed in this paper, in which the geometric structure of multi-point compact is analyzed, and the mathematical model of geometric constraints is established, and the matching relationship of the whole assembly is also analyzed. In view of the lack of positioning datum on the cabin, this paper analyzes the datum assembly form in the assembly process, and establishes the datum transfer and coordinate system establishment scheme, and then gives the calculation method of gasket compensation. The method proposed in this paper is helpful to improve the assembly and adjustment of multi-point pressing type, ensure the assembly quality and improve the assembly efficiency, which can provide reference for other similar space equipment by using digital assembly methods.

\section{References}

1. Binglong $\mathrm{W}$, Daokui Q, Fang X. Industrial Robot High Precision Peg-in-hole Assembly based on Hybrid Force/Position Control. J. Journal of Zhejiang University (Engineering Science), 52, 379(2018)

2. Mei Z, Maropoulos P G. Review of the Application of Flexible, Measurement-assisted Assembly Technology in Aircraft Manufacturing. J. Proceedings of the Institution of Mechanical
Engineers, Part B: Journal of Engineering Manufacture, 228, 1185(2014)

3. Yuan L, Li Z, Yanzhong W. An Optimal Method of Posture Adjustment in Aircraft Fuselage Joining Assembly with Engineering Constraints. J. Chinese Journal of Aeronautics, 30, 2016(2017)

4. Junxiu L, Zongquan D, Honghao Y. Research on locked point arrangement method of spatial mechanical arm. J. Jouenal of Machine Design, 29, 85(2012)

5. Zongquan D, Binghui W, Xiang W. Design and analysis of anew style lock-unlock mechanism for spaceborne appendages. C. Proceedings of the International conference on Advanced Design and manufacture, 117(2006)

6. Jun W. Research on Large Load and Distributed Locking-releasing Mechanism of Space Manipulator. D. Harbin Institute of Technology, (2014)

7. Graham G, External R, Savi S, et al. Canada and the international space station program: overview and status. J. Acta Astronautica, 51, 59(2002)

8. Heemskerk C J M, Schoonejans P H M. Overview of software engineering applications in the european robotic arm. C. Proceedings of the NASA 97 Conference on "Data System in Aerospace" , 317(1997)

9. Huifang Z, Zongquan D, Xiang W, et al. Kinematic Analysis of releasing process of Locking- releasing Mechanism for Robotic Arm. J. Mechanical Engineer, 49(2006) 